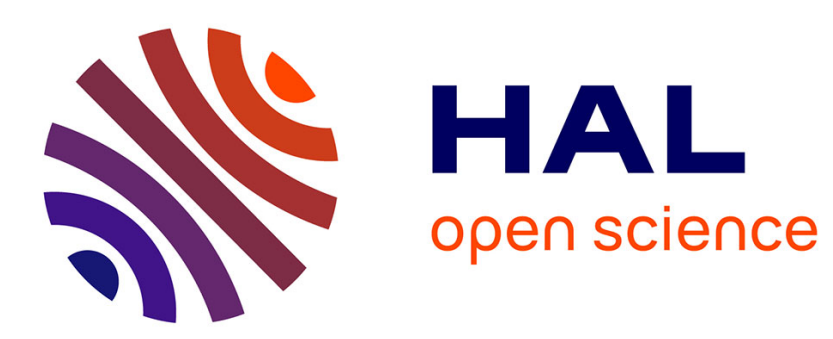

\title{
Modal parameter identification by an iterative approach and by the state space model
}

Joseph Lardies

\section{To cite this version:}

Joseph Lardies. Modal parameter identification by an iterative approach and by the state space model. Mechanical Systems and Signal Processing, 2017, 95, pp.239-251. 10.1016/j.ymssp.2017.03.010 . hal02131279

\section{HAL Id: hal-02131279 \\ https://hal.science/hal-02131279}

Submitted on 16 May 2019

HAL is a multi-disciplinary open access archive for the deposit and dissemination of scientific research documents, whether they are published or not. The documents may come from teaching and research institutions in France or abroad, or from public or private research centers.
L'archive ouverte pluridisciplinaire HAL, est destinée au dépôt et à la diffusion de documents scientifiques de niveau recherche, publiés ou non, émanant des établissements d'enseignement et de recherche français ou étrangers, des laboratoires publics ou privés. 


\title{
MODAL PARAMETER IDENTIFICATION BY AN ITERATIVE APPROACH AND BY THE STATE SPACE MODEL
}

\author{
Joseph LARDIES \\ University of Bourgogne Franche-Comté \\ FEMTO-ST Institute \\ CNRS/UFC/ENSMM/UTBM \\ Department of Applied Mechanics \\ 25000 Besançon; FRANCE
}

Email : joseph.lardies@univ-fcomte.fr 


\begin{abstract}
The problem of estimating a spectral representation of exponentially decaying signals from a set of sampled data is of considerable interest in several applications such as in vibration analysis of mechanical systems. In this paper we present a nonparametric and a parametric method for modal parameter identification of vibrating systems when only output data is available. The nonparametric method uses an iterative adaptive algorithm based in the formation of a two dimensional grid mesh, both in frequency and damping domains. We formulate the identification problem as an optimization problem where the signal energy is obtained from each frequency grid point and damping grid point. The modal parameters are then obtained by minimizing the signal energy from all grid points other than the grid point which contains the modal parameters of the system. The parametric approach uses the state space model and properties of the controllability matrix to obtain the state transition matrix which contains all modal information. We discuss and illustrate the benefits of the proposed algorithms using a numerical and two experimental tests and we conclude that the nonparametric approach is very time consuming when a large number of samples is considered and does not outperform the parametric approach.
\end{abstract}

Key-words: Modal parameters, iterative algorithm, state space model, controllability matrix, transition matrix 


\section{INTRODUCTION}

In many applications the mechanical structure under consideration is excited by a brief input signal, called an impulse. This is particularly so in mechanical vibration analysis test situations, where the test system is excited by a force acting over a very short duration time: for example, the system is struck by a hammer. Other common types of vibration testing services are conducted using a step force which is constant through a time frame. The corresponding response of the system to an impulse or a step force is a transient response, since steady-state oscillations are not produced and oscillations of the system reduce to zero after a finite time. Consequently, transient responses are short time events whose time behavior cannot be predicted and are totally varying in nature, both in time and frequency. Two fundamental parameters to identify from the transient response of a vibrating system are the eigenfrequencies and the damping coefficients and we are faced to a problem of spectral analysis. The problem of estimating the spectral parameters of damped signals has attracted significant attention during recent decades as such signals arise naturally in many engineering domains. We can mention the vibration monitoring [1, 2], where the spectral content of measured signals gives information on the wear of mechanical parts under study, the magnetic resonance spectroscopy [3] to obtain further information about cellular activity, in speech analysis [4] for speech synthesis and speech recognition, in geophysical seismology [5] to predict subsurface geologic structure, in sonar and in radar [6] to estimate the locations and waveforms of acoustical or electromagnetic sources. Nonparametric and parametric spectral estimators are usually used [7]. A nonparametric estimator attempts to compute the spectral content of a signal without using any a priori information about it: there is no assumption about how the data is generated. The most common nonparametric estimator is the traditional periodogram which basically reduces to the computation of a Discrete Fourier Transform and gives a power spectral density estimate of the signal. Bartlett, Daniell and Welch [7] have proposed refined periodograms by smoothing or averaging the traditional periodogram obtained from segments which are windowed. Recently, Gudmundson [8, 9] has implemented an iterative adaptive approach for radiofrequency spectroscopy in the case of irregularly sampled data. The algorithm uses data from nuclear quadrupole resonance experiments and is employed to determine ammonium nitrate and cyclonite in a liquid. 
A parametric or model-based estimator assumes that the structure of the signal is known: the signal satisfies a generating model with known functional form. Parametric methods are used to estimate the parameters in the assumed model and the most common of the parametric estimation technique is the auto-regressive moving average (ARMA) modeling of the signal [10]. The modal parameters are obtained from eigendecomposition of the companion matrix which is formed with the AR coefficients. The ARMA approach, unlike the nonparametric approaches, requires the selection of the order, or the structure, of the model. The selection of model order is a problem always under investigation. Other parametric methods using subspace algorithms and derived from the state space model have been developed and are always part of an intense research [11-15]. Note that none of these parametric methods is able to cope with very low snapshot numbers or with a severe measurement noise. The problem of measurement noise has been investigated by J.N. Juang and R.S. Papa in [16] where the errors characteristics on modal parameters due to noise have been analyzed by numerical simulations using the Monte Carlo technique and the eigensystem realization algorithm. More recently S. Dorvash and S.N. Pakzad [17] have presented the influence of measurement noise on modal parameter identification using experimental tests and the eigensystem realization algorithm. It is shown that the deviation of the modal parameters obtained by the low-noise sensors is generally less than the deviation of the modal parameters identified by the sensors with higher noise level. The authors concluded that the attenuation of this deviation can be performed by increasing the model order of the system and through the use of a stabilization diagram.

To estimate the eigenfrequencies and damping coefficients of a vibrating system from output data only we propose a nonparametric iterative adaptive algorithm and a parametric approach based on a subspace algorithm. The iterative adaptive technique does not require the specification of any user parameters. Furthermore, no assumptions have to be made on the sampling scheme and the spectrum can be estimated from regularly or even irregularly sampled data [3]. To our knowledge it is the first time that the iterative adaptive method is applied to modal parameter identification of mechanical systems and constitutes a novelty. Furthermore, this algorithm uses a two-dimensional grid mesh, both in frequency and damping domain and a strategy to choose the grid mesh is presented.

The proposed subspace algorithm, which is derived from the state space model, is based on shifted properties of the controllability matrix. The matrices operations that yield from the controllability matrix to the state transition matrix are a peculiarity of the paper. To illustrate the effectiveness of the proposed algorithms, we examine the results on simulated data from 
exponentially damped sinusoids corrupted by white noise, on experimental measurements from the displacement of a micro electro mechanical system (MEMS) constituted of a perforated microplate and on measurements from the vibrations of a line cable excited through an impact hammer. Note that to our knowledge it is the first time that the proposed subspace algorithm is used to identify the modal parameters of a MEMS and one of the goals of this work is to compare the iterative adaptive algorithm and the subspace algorithm using numerical and experimental tests.

The paper is outlined as follows. In the next section we present the data model and derive a nonparametric approach based on the iterative adaptive algorithm for modal parameter identification. In section 3 we propose a parametric approach derived from the state space model with a subspace algorithm. The performances of the proposed methods are studied in section 4 using both simulated and measured data obtained from the movement of a perforated microplate and from the vibrations of a line cable. This paper is briefly concluded in section 5 .

\section{THE ITERATIVE ADAPTIVE ALGORITHM IN MECHANICAL VIBRATIONS}

\subsection{Response of a MDOF system}

The linear MDOF system is governed by the general equation

$$
\boldsymbol{M} \ddot{Z}(t)+C \dot{Z}(t)+\boldsymbol{K} \boldsymbol{Z}(t)=\boldsymbol{F}
$$

where $\boldsymbol{M}, \boldsymbol{C}$, and $\boldsymbol{K}$ are mass, damping and stiffness matrices respectively, and $\boldsymbol{F}$ is the excitation vector. The response $\boldsymbol{Z}$ of the system can be obtained using well known modal analysis or a direct forced response method. Instead of the MDOF system given by (1), $N$ uncoupled equations similar to a SDOF system can be obtained

$$
\mathrm{m}_{\mathrm{i}} \ddot{z}(t)+c_{i} \dot{z}(t)+k_{i} z(t)=f_{i}(t)
$$

for $i=1,2 \ldots, N$. 
The impulse response of this MDOF system is obtained as the superposition of the $N$ relevant modes

$$
h(t)=\sum_{i=1}^{N} A_{i} e^{\left(-\xi_{i} \omega_{n i} t\right)} \cos \left(\omega_{d i} t+\varphi_{i}\right)
$$

where for the $i^{\text {th }}$ mode $\omega_{n i}=2 \pi F_{n i}$ is the natural or undamped frequency in rad.s ${ }^{-1}\left(F_{n i}\right.$ is the $i^{\text {th }}$ natural frequency in $\left.\mathrm{Hz}\right), \xi_{i}$ is the $i^{\text {th }}$ damping ratio and $\omega_{d i}$ is the $i^{\text {th }}$ damped natural frequency $\left(\omega_{d i}=\omega_{n i} \sqrt{1-\xi_{i}^{2}}\right), A_{\mathrm{i}}$ is the $i^{\text {th }}$ residue magnitude and $\varphi_{i}$ is the $i^{\text {th }}$ phase lag. In many practical vibrating systems we have $\xi_{i}<<1$, so we shall consider $\omega_{d i} \approx \omega_{n i}$. This response is a linear combination of single modal components and each mode is given by an exponentially decaying harmonic function. In complex notation, this equation can be represented as a sum of $N$ complex exponentially damped sinusoidal components (or damped sinusoids) and if we add a measurement noise $e(t)$ we obtain:

$$
y(t)=\sum_{i=1}^{N} A_{i} e^{\left[-\xi_{i} \omega_{n i} t+j\left(\omega_{d i} t+\varphi_{i}\right)\right]}+e(t)
$$

If we set $\alpha_{i}=\xi_{i} \omega_{n i}$ and $B_{i}=A_{i} e^{j \varphi_{i}}$ (4) can be written as

$$
y(t)=\sum_{i=1}^{N} B_{i} e^{\left(-\alpha_{i}+j \omega_{d i}\right) t}+e(t)
$$

The additive noise is assumed to be zero mean, Gaussian with variance $\sigma$ and uncorrelated with the damped sinusoids. Note that the noise component can be omitted in the model and can be included implicitly via $B_{i}$. Given the knowledge of the response $y(t)$, the goal is to identify the decay rate $\alpha_{i}$ and the damped natural frequency $\omega_{d i}$ for each mode from the impulse response of a MDOF system. We can then readily obtain the damping ratio $\xi_{i}$ and the natural frequency $\omega_{n i}$ :

$$
\xi_{i}=\frac{\alpha_{i}}{\sqrt{\alpha_{i}^{2}+\omega_{d i}^{2}}} \text { and } \omega_{n i}=\frac{\omega_{d i}}{\sqrt{1-\xi_{i}^{2}}} \approx \omega_{d i}
$$




\subsection{The iterative adaptive algorithm}

In this section we propose a nonparametric iterative adaptive algorithm based in the formation of a grid mesh in frequency and damping domains and in the estimation of the signal energy at each grid point. An original strategy for multiresolution grid refinement is described at the end of this section.

Let $\boldsymbol{y}=\left[\begin{array}{llll}y_{t 1} & y_{t 2} & \ldots & y_{t T}\end{array}\right]^{\mathrm{T}}$ be the vector of available measurements, ()$^{\mathrm{T}}$ denotes the transpose operation and $\left\{t_{p}\right\}_{p=1}^{T}$ the sampling times of $T$ available samples. Let $K$ denote the number of grid points in the damped frequency domain and let $\left\{\omega_{d k}\right\}_{k=1}^{K}$ be the corresponding damped frequencies. Usually $K$ is chosen to be quite large $(\mathrm{K}>>\mathrm{N})$ and the damped frequency grid of interest $\left\{\omega_{d k}\right\}$ is uniform. Let $M$ denote the number of grid points in the decay rate domain $(M>>N)$ and let $\left\{\alpha_{m}\right\}_{m=1}^{M}$ be the corresponding decay rates. We will now rewrite the response (5) as the sum of the contributions from each damped frequency grid point and each decay rate grid point

$$
\mathrm{y}_{t p}=\sum_{k=1}^{K} \sum_{m=1}^{M} B_{k, m} e^{\left(-\alpha_{m}+j \omega_{d k}\right) t p}+e_{t p}
$$

where $B_{k, m}$ represents the amplitude for a possible damped sinusoidal component (or a grid point), with damped frequency $\omega_{d k}$ and decay rate $\alpha_{m}$. In our framework, the estimates of modal parameters are confined to a scanning grid. We cannot make the grid very fine since this would increase the computational complexity significantly. We explore the idea of adaptively refining the grid in order to achieve better precision. The idea is a very natural one: instead of having a universally fine grid, we make the grid fine only around the regions where the modes are present.

We denote the damped Fourier vector for damped frequency $\omega_{d k}$ and decay rate $\alpha_{m}$ as

$$
\boldsymbol{a}_{k, m}=\left[e^{\left(-\alpha_{m}+j \omega_{d k}\right) t}{ }_{1} e^{\left(-\alpha_{m}+j \omega_{d k}\right) t}{ }_{2} \ldots e^{\left(-\alpha_{m}+j \omega_{d k}\right) t} t_{T}\right]^{\mathrm{T}}
$$


and the data vector takes the form

$$
\boldsymbol{y}=\sum_{k=1}^{K} \sum_{m=1}^{M} B_{k, m} \boldsymbol{a}_{k, m}+\boldsymbol{e}
$$

where $\boldsymbol{e}$ is defined similarly to $\boldsymbol{y}$. Denote the signal energy at the grid point $\left(\omega_{d k}, \alpha_{m}\right)$ as $p_{k, m}=\left|B_{k, m}\right|^{2}$. This value is small except for a number of grid points ( $N$ grid points) corresponding to the positions of the true modal parameters and our objective is to find these grid points. The covariance matrix of the vector $\boldsymbol{y}$ is defined as:

$$
\boldsymbol{R}=\sum_{k=1}^{K} \sum_{m=1}^{M} p_{k, m} \boldsymbol{a}_{k, m} \boldsymbol{a}_{k, m}^{*}+\sigma \boldsymbol{I}
$$

Where the superscript $*$ indicates the complex conjugate transposition. In order to find an estimate of $B_{k, m}$ we consider the general linear estimator: $\hat{B}_{k, m}=\boldsymbol{w}_{k, m}{ }_{k, m} \boldsymbol{y}$. The weight vector $\boldsymbol{w}_{k, m}$ can be found by minimizing the power from all grid points other than the grid point $\left(\omega_{d k}\right.$, $\alpha_{m}$ ) which passes the component of interest undistorted: the true modes of the vibrating system are perfectly reproduced in appropriate grid points. We have then the following minimization problem:

$$
\min \left(\boldsymbol{w}_{k, m}^{*} \boldsymbol{R} \boldsymbol{w}_{k, \boldsymbol{m}}\right) \quad \text { subject to the constraint } \quad \boldsymbol{w}_{k, m}^{*} \boldsymbol{a}_{k, \boldsymbol{m}}=1
$$

It is shown in Appendix that :

$$
w_{k, m}=\frac{R^{-1} a_{k, m}}{a_{k, m}^{*} R^{-1} a_{k, m}}
$$

and an estimate of the amplitude at the grid point $\left(\omega_{d k}, \alpha_{m}\right)$ is

$$
\hat{B}_{k, m}=\frac{a^{*} k, m R^{-1} y}{a_{k, m}^{*} R^{-1} a_{k, m}}
$$

As the covariance matrix $\boldsymbol{R}$ depends on the estimates of the amplitudes at each grid point (13) must be implemented as an iterative algorithm. The initial estimator is obtained with $\boldsymbol{R}=\boldsymbol{I}$, 
the identity matrix. The iterative adaptive algorithm for spectral estimation is thus found by iterating the estimation of $\boldsymbol{R}$ in (10) and the grid point amplitudes in (13) until a suitable stop criterion is met. The procedure is as follows: let $\hat{B}_{k, m}^{(i+1)}$ denote the estimate of the amplitude at iteration $i+1$ and $\boldsymbol{R}_{(i)}$ the matrix formed from (10) with $\hat{B}_{k, m}^{(i)}$ (or $p_{k, m}^{(i)}$ ), then the iterative adaptive algorithm updates the estimates as:

$$
\hat{B}_{k, m}^{(i+1)}=\frac{\boldsymbol{a}^{*} \boldsymbol{k}, \boldsymbol{m} \boldsymbol{R}_{(i)}^{-1} \boldsymbol{y}}{\boldsymbol{a}_{\boldsymbol{k}, \boldsymbol{m}}^{*} \boldsymbol{R}_{(i)}^{-1} \boldsymbol{a}_{\boldsymbol{k}, \boldsymbol{m}}} \quad i=1,2 \ldots
$$

The iterative process will be stopped when the relative change in the estimated amplitude $\hat{B}_{k, m}$ i.e. $\left|\hat{B}_{k, m}^{(i+1)}-\hat{B}_{k, m}^{(i)}\right|^{2}<10^{-3}$. It has been found that the algorithm does not provide significant improvements in performance after about 10 iterations. Table 1 provides a summary of the required steps used in the iterative adaptive algorithm.

The estimates are confined to a scanning grid and we make the grid fine only around the regions where the two modal parameters are present. This requires an approximate knowledge of the locations of the modes, which can be obtained by using a coarse grid first or from the periodogram. We have the following procedure:

- Create a rough grid of potential modes

- Compute the signal energy estimate $\hat{p}_{k, m}$ (step 2 of the algorithm given in Table 1)

- Compute the estimated covariance matrix (step 3 of the algorithm given in Table 1)

- Compute the amplitude estimate $\hat{B}_{k, m}$ (step 4 of the algorithm given in Table 1)

- Get a refined grid around the locations of the modes

Many different ways to refine the grid can be imagined, however, we choose simple equispaced grid refinement. The iterative process is stopped by the residual amplitude threshold: the difference between the amplitudes given in step 4 of the algorithm or (14) are smaller than a threshold (see Table 1). It has been found that iterating the algorithm 10 times is generally enough to give a good solution.

Numerical and experimental examples are shown in section 4 to illustrate the performances of the proposed algorithm. 


\section{THE STATE SPACE MODEL}

In this section we propose a parametric approach based in the state space model and the use of a subspace algorithm for modal parameter identification. The key point of the subspace algorithm is the determination of the state transition matrix which contains all modal information. This state transition matrix is obtained using shifting properties of the controllability matrix, and this approach is used from experimental measurements in time domain, for modal parameter identification of a MEMS. In the state space model the covariance matrices of noisy data are given by [11-14]

$$
\boldsymbol{R}_{\boldsymbol{i}}=\boldsymbol{C A} \boldsymbol{A}^{i-1} \boldsymbol{G}
$$

where $(\boldsymbol{A}, \boldsymbol{C}, \boldsymbol{G})$ are the state space parameters of the system [11,13]. The eigenvalues of the $N \mathrm{x} N$ state transition matrix $\boldsymbol{A}$ are equal to $z_{i}=e^{\left(-\alpha_{i}+j \omega_{d i}\right)}$, the parameters of interest, and our objective is to estimate the transition matrix. In order to estimate the matrix $\boldsymbol{A}$ we form the Hankel matrix $\boldsymbol{H}$ with two factorizations: the first factorization uses the observability and controllability matrices, $\boldsymbol{O}$ and $\boldsymbol{K}$, as:

$$
H=\left[\begin{array}{cccc}
R_{1} & R_{2} & \cdot & R_{p} \\
R_{2} & R_{3} & \cdot & R_{p+1} \\
\cdot & \cdot & \cdot & \cdot \\
R_{f} & R_{f+1} & \cdot & R_{f+p-1}
\end{array}\right]=\left[\begin{array}{c}
C \\
C A \\
\cdot \\
C A^{f-1}
\end{array}\right]\left[G A G . . A^{p-1} G\right]=O K
$$

where $\boldsymbol{O}$ is the observability matrix and $\boldsymbol{K}$ is the controllability matrix :

$$
\boldsymbol{O}=\left[\begin{array}{c}
C \\
C A \\
\cdot \\
C A^{f-1}
\end{array}\right] \quad ; \quad K=\left[G A G . \ldots A^{p-1} G\right]
$$

The second factorization uses the singular value decomposition (SVD) of $\boldsymbol{H}$

$$
H=U \Sigma V^{T}=U \Sigma^{1 / 2} \Sigma^{1 / 2} V^{T}
$$


with $\boldsymbol{U}^{T} \boldsymbol{U}$ and $\boldsymbol{V}^{T} \boldsymbol{V}$ identity matrices and $\Sigma$ a diagonal matrix of singular values.

The two factorizations of the block Hankel matrix are equated to give

$$
H=U \Sigma^{1 / 2} \Sigma^{1 / 2} V^{T}=O K
$$

implying $\boldsymbol{O}=\boldsymbol{U} \Sigma^{1 / 2}$ and $\boldsymbol{K}=\Sigma^{1 / 2} \boldsymbol{V}^{\boldsymbol{T}}$. To determine the transition matrix $\boldsymbol{A}$ we use properties of the controllability matrix (properties of the observability matrix can also be used). We introduce the two following matrices:

$$
K \Uparrow=\left[\begin{array}{ll}
A G & A^{2} G \ldots . . A^{p-1} G
\end{array}\right] \text { and } K \Downarrow=\left[\begin{array}{lll}
G & A G & A^{2} G \ldots . . A^{p-2} G
\end{array}\right]
$$

where $\boldsymbol{K} \Uparrow$ is the matrix obtained by deleting the first block column of $\boldsymbol{K}$ and $\boldsymbol{K} \Downarrow$ is the matrix obtained by deleting the last block column $\boldsymbol{K}$. We obtain then:

$$
K \Uparrow=A K \Downarrow \text { or } \quad\left(\Sigma^{1 / 2} V^{T}\right) \Uparrow=A\left(\Sigma^{1 / 2} V^{T}\right) \Downarrow
$$

We use properties of shifting columns operators " $\Uparrow^{\prime \prime}$ and $"{ }_{\Downarrow}, "$ : let $\boldsymbol{\Phi}$ and $\boldsymbol{\Psi}$ be two (axb) and $(b x c)$ matrices, we have : $(\boldsymbol{\Phi} \boldsymbol{\Psi}) \Uparrow=(\boldsymbol{\Phi} \boldsymbol{\Psi} \Uparrow)$ and $(\boldsymbol{\Phi} \boldsymbol{\Psi}) \Downarrow=(\boldsymbol{\Phi} \boldsymbol{\Psi} \Downarrow)$ consequently

$$
\left(\Sigma^{1 / 2} V^{T} \Uparrow\right)=A\left(\Sigma^{1 / 2} V^{T} \Downarrow\right)
$$

The transition matrix is

$$
A=\left(\Sigma^{1 / 2} V^{T} \Uparrow\right)\left(\Sigma^{1 / 2} V^{T} \Downarrow\right)^{+}
$$

where ()$^{+}$represents the pseudo inverse of a matrix, and the eigenvalues of the transition matrix are given by

$$
\lambda(A)=\lambda\left[\left(V^{T} \Uparrow\right)\left(V^{T} \Downarrow\right)^{+}\right]
$$

This approach constitutes a subspace modal identification method and in the case of noisy data a problem of model order determination occurs: when extracting physical modes this algorithm can generate spurious modes. For this reason, the assumed number of modes, or model order, is incremented over a wide range of values and we plot the stability diagram. 
The stability diagram involves tracking the estimates of eigenfrequencies and damping ratios as a function of model order $[14,15]$. As the model order is increased, more and more modal frequencies and damping ratios are estimated, hopefully, the estimates of the physical modal parameters stabilize using a criterion based on the modal coherence of measured and identified modes [14].

\section{APPLICATIONS}

\subsection{Simulated data}

We illustrate the performances of proposed algorithms using simulated data. We generate data containing three exponentially damped sinusoids and a harmonic component. The normalized frequencies are $0.1 ; 0.2 ; 0.3$ and 0.4 and the decay rates $\alpha_{i}$ are $0.03 ; 0.04 ; 0.05$ and 0 . We have to identify three structural modes and a harmonic component. The data was corrupted using a complex white Gaussian noise and the SNR is equal to $3 \mathrm{~dB}$ or $10 \mathrm{~dB}$. The problem to distinguish harmonic and structural components has been analyzed by T-P Le and P. Argoul [18] using a time-frequency decomposition. This lead to the computation of the kurtosis and histograms corresponding to the ridges of the wavelet transform. The procedure for the distinction between such components is very time consuming and the technique is extremely cumbersome. The two methods presented in the paper can be used to obtain directly the distinction between harmonic and structural components. In our numerical test, only 30 time samples are used for the identification by nonparametric approaches. In first, we compare the traditional periodogram spectrum and the Welch spectrum with the spectrum of the iterative adaptive algorithm. The periodogram spectrum is commonly used for estimation of dampings and eigenfrequencies where the dampings can be estimated from half the width of the peaks at half their heights. Figures 1 and 2 show the traditional periodogram and the Welch spectrum with $\mathrm{SNR}=3 \mathrm{~dB}$ or $\mathrm{SNR}=10 \mathrm{~dB}$ : from these plots it is very difficult to identify the damping coefficients, furthermore some spurious modes appear and the harmonic component has a damping coefficient. These nonparametric estimators fail in the procedure of modal parameter identification. To improve the results the iterative adaptive algorithm and the subspace algorithm are proposed.

Figure 3 shows the iterative adaptive spectrum with $S N R=10 \mathrm{~dB}$ for different iterations. We present the evolution of the spectrum after one iteration, five iterations and ten iterations. After ten iterations the modal parameters are well identified as shown in Figure 4 with the contour plot. No spurious modes appear and we can differentiate the damped sinusoids and 
the harmonic component. Even with a low SNR of $3 \mathrm{~dB}$ we can identify the modal parameters. Note that with ten iterations the computation time is a few minutes.

Subspace algorithms have a problem of model order determination. When extracting physical or structural modes, this time domain modal identification algorithm always generate spurious or computational modes to account for unwanted effects such as noise, leakage, residuals, non-linearity's...A modal indicator to distinguish structural and spurious modes has been proposed in [14] and is used in the paper. Figure 5 shows the stabilization diagram on eigenfrequencies and damping ratios. We have used 150 time samples and the computation time is a few seconds. The true damping ratios are $4.7 \% ; 3.18 \% ; 2.65 \%$ and $0 \%$ and are well identified from these stabilization diagrams. A different color is used for each mode and the color is associated in each pair of stabilization diagrams, for eigenfrequencies and damping ratios. Note that the stabilization diagrams are plotted only for model orders higher than about 20. Indeed, the modal indicator used to distinguish structural and computational or spurious modes may fail for very low model orders because the true modes can be considered as spurious and disappear of the stabilization diagram.

\subsection{Modal parameter identification of a perforated microplate}

The design of micro electro mechanical systems (MEMS) includes oscillating elements which are often perforated microplates supported by elastic suspensions. These microplates must be involved for MEMS applications as switches, varactors, transceivers, radio frequency devices and energy harvesters. The study of the damping caused by the surrounding fluid and by the dissipation in the material is very important to predict the dynamic response of the structure and to estimate some important parameters such as the quality factor, the release time and the switching time [19]. A single degree of freedom model is generally suitable to study such microplates with elastic supports, because the mass is concentrated in the central plate and the suspensions correspond to the elastic deformable part. A traditional spring-massdamper mechanical model is used to describe the comportment of a microplate and the model is then defined by the following parameters to identify: the microplate mass $m_{p}$, the stiffness (or elastic coefficient) $k_{p}$ and the damping ratio $\xi$ of the system.

Figure 6 shows a schematic of the perforated microplate where the geometrical dimensions are: microplate side $a=185.96 \mu \mathrm{m}$, microplate thickness $h_{c}=6.312 \mu \mathrm{m}$, gap thickness $h=3 \mu \mathrm{m}$, hole size $s_{0}=7.19 \mu \mathrm{m}$, microplate density $\rho=19.32 \times 10^{3} \mathrm{~kg} \cdot \mathrm{m}^{-3}$. The number of holes is $N_{h}=64$. The electrode area is $A=a^{2}-N_{h} s_{0}=3.127 \times 10^{-8} \mathrm{~m}^{2}$ and the 
microplate mass is $m=\rho A h_{c}=3.81 \times 10^{-9} \mathrm{~kg}$. Our objective is to determine the plate stiffness $k_{p}$ from the resonance frequency $F_{r}$ (we have the following relation $\left.k_{p}=m\left(2 \pi F_{r}\right)^{2}\right)$ and the damping ratio $\xi$ using algorithms developed in the paper. The dynamic measurements are conducted in the time domain by means of a laser vibrometer. The time response of the microplate to a step actuation force is given in Figure 7 and the sampling frequency of signals is $2 \mathrm{MHz}$. Using only a part of this response (3000 time samples), the iterative adaptive algorithm is then applied and we obtain the spectrum and the contour plots given in Figures 8 and 9. We can then deduce the resonance frequency: $F_{r}=2.28 \times 10^{4} \mathrm{~Hz}$, the microplate stiffness $k_{p}=78.25 \mathrm{~N} \cdot \mathrm{m}^{-1}$, the decay rate $\alpha=2 \pi \times 342$ and the damping ratio of the system $\xi=\alpha / 2 \pi F_{r}=0.015$.

Using the subspace algorithm developed in the paper with 3000 data points to form autocovariance matrices, we plot in Figure 10 the stabilization diagram on eigenfrequencies and damping ratios for the experimental system. The resonance frequency of the vibrating microplate is $F_{r}=2.28 \times 10^{4} \mathrm{~Hz}$ and the damping ratio is $\xi=0.0164$. These modal parameters have been obtained by an average over the orders of stabilization diagrams and the results are comparable to those obtained by the iterative adaptive algorithm. To our knowledge, it is the first time that the iterative adaptive algorithm and the subspace algorithm are applied to a micro electro mechanical system and in particular to a microplate. Note that the iterative adaptive algorithm is very time consuming compared with the subspace algorithm where only a few seconds are needed for the identification.

\subsection{Modal parameter identification of a line cable in laboratory}

For validation of the proposed algorithms another experimental test is performed in laboratory with a horizontal line cable excited through an impulse. The length of the cable is 32.3 meters and the excitation is obtained through an impact hammer at 5.38 meters of the extremity of the cable. The experimental time data are acquired using accelerometers placed along the cable. The recording time is $32 \mathrm{~s}$ and the sampling frequency is $64 \mathrm{~Hz}$. We have then 2048 time samples available. Details on the experimental procedure and results obtained by another subspace algorithm and by the wavelet transform can be found in [14]. Figures 11 and 12 show the spectrum and the contour of the iterative adaptive algorithm using time data of an accelerometer. The modal parameters of the cable are then obtained and are presented in Table 2. 
Figure 13 shows the stabilization diagram on eigenfrequencies and damping ratios. The stabilization diagram shows very stable eigenfrequencies and stable damping ratios, and from this plot we obtain the experimental eigenfrequencies and damping ratios of the cable by averaging the values obtained from different orders. Table 2 shows the theoretical and experimental natural frequencies and damping ratios of the cable using the methods presented in the paper. The results obtained by the iterative adaptive algorithm and by the subspace method are comparable, however the iterative adaptive algorithm is very time consuming.

\section{CONCLUSION}

The problem of estimating the modal parameters of vibrating systems from output data only attracts significant attention as such parameters can be used for fault detection, structural health monitoring and model validation. These modal parameters have been identified using two approaches: a nonparametric adaptive iterative algorithm and a parametric subspace algorithm. The spectrum contour plot of the adaptive algorithm gives the eigenfrequencies and damping ratios of vibrating systems. Using the subspace algorithm, the stabilization diagram on eigenfrequencies and damping ratios gives directly the modal parameters. The proposed methods outperform the commonly used periodogram method and display similar results for the extracted modal parameters. Damped modes and harmonic components can be identified using the two developed algorithms. The principal advantages of the iterative adaptive algorithm are:

- it does not require the specification of any user parameters, in particular it is not necessary to know (or to find) the model order of the system

- it has better performances than the classical periodogram algorithm

- $\quad$ it yields an accurate 3D (amplitude, frequency and damping) spectral representation and we can identify simultaneously eigenfrequencies and damping ratios from this representation.

However, with a large number of samples the benefits of the iterative algorithm are restricted: this algorithm is very time consuming, since additional computational cost is necessary due to the inversion of the sample covariance matrix and the use of iterations, so this algorithm does not outperform the subspace algorithm. It will be interesting to optimize this algorithm in 
order to obtain a reduction of the computational complexity without parameter identification degradation. Furthermore, it is necessary to note that the iterative adaptive approach can be applied only when exponentially decaying signals are considered, whereas the subspace algorithm does not suffer this limitation.

The algorithms presented in the paper have been originally tested for the modal parameter identification of a micro electro mechanical structure and are an alternative to wavelet transform algorithms which constitute a time-frequency representation and are currently used in modal parameter identification [18]. It will be interesting to compare the results obtained by the two approaches developed here with the wavelet transform method in particular for the analysis of micro electro mechanical systems. The algorithms developed in the paper can be used easily in order to differentiate between harmonic and structural components. 


\section{Appendix}

We have then the following minimization problem:

$\min \left(\boldsymbol{w}_{k, m}^{*} \boldsymbol{R} \boldsymbol{w}_{\boldsymbol{k}, \boldsymbol{m}}\right) \quad$ subject to the constraint $\quad \boldsymbol{w}^{*}{ }_{k, m} \boldsymbol{a}_{k, \boldsymbol{m}}=1$

Using the Langrange's method we can minimise the power subject to this constraints by defining the cost function

$H\left(\boldsymbol{w}^{*}{ }_{k, m}\right)=\boldsymbol{w}_{k, m}^{*} \boldsymbol{a}_{k, \boldsymbol{m}}+2 \lambda\left(\boldsymbol{w}_{k, m}^{*} \boldsymbol{a}_{k, \boldsymbol{m}}-1\right)$

where $\lambda$ is a Lagrange multiplier.

The optimal value is obtained when $\boldsymbol{g r a d}\left(H\left(\boldsymbol{w}_{k, m}^{*}\right)\right)=2 \boldsymbol{R} \boldsymbol{w}_{k, m}+2 \lambda \boldsymbol{a}_{k, \boldsymbol{m}}=\mathbf{0}$

$\boldsymbol{w}_{k, m}=-\lambda \boldsymbol{R}^{-1} \boldsymbol{a}_{k, \boldsymbol{m}}$. Using the constraint we deduce $\lambda=-\left(\boldsymbol{a}_{k, m}^{*} \boldsymbol{R}^{-\boldsymbol{1}} \boldsymbol{a}_{k, \boldsymbol{m}}\right)^{-1}$

and the optimum weight vector is $\quad w_{k, m}=\frac{R^{-1} a_{k, m}}{a_{k, m}^{*} R^{-1} a_{k, m}}$ 


\section{REFERENCES}

1.S.G. BRAUN Mechanical Signature Analysis. Theory and Applications, 1986, Academic Press.

2. M.D. SPIRIDONAKOS and S.D. FASSOIS 2009 Mechanical Systems and Signal Processing 23, pp. 2029-2048. Parameter identification of a time-varying structure based on vector vibration response measurements.

3. E. GUDMUNDSON 2010 Signal Processing for Spectroscopic Applications, PHD. Thesis, Uppsala University, Sweden.

4. S. FURUI Digital Speech Processing, Synthesis and Recognition, 1989, Marcel Dekker.

5. J. HAN and M. VAN DER BAAN 2013 Geophysics,78, pp. 9-19. Empirical mode decomposition for seismic time-frequency analysis.

6. R.O. SCHMIDT 1986 IEEE Transactions on Antennas and Propagation,34, pp.276-280. Multiple emitter location and signal parameter estimation.

7. P. STOICA and R. MOSES Spectral Analysis of Signals, 2005, Prentice Hall.

8. E. GUDMUNDSON and A. JAKOBSSON 2007 Proceedings of the $41^{\text {st }}$ Asilomar Conference on Signals, Systems and Computers, California, USA. Efficient algorithms for computing the Capon and APES Filters.

9. E. GUDMUNDSON, P. STOICA, J. LI, A. JAKOBSSON, M.D. ROWE, J.A.S. SMITH, J. LUNG 2010 Journal of Magnetic Resonance 203, pp.167-176. Spectral estimation of irregularly sampled exponentially decaying signals with applications to RF spectroscopy.

10. K.A. PETSOUNIS and S.D. FASSOIS 2001 Mechanical Systems and Signal Processing 15, pp. 1031-1060. Parametric time-domain methods for the identification of vibrating structures- a critical comparison and assessment. 
11. P. VAN OVERSCHEE and B. DE MOOR 1996 Subspace Identification for Linear Systems : Theory-Implementations-Methods. Kluwer Academic.

12. J.N. JUANG 1994 Applied System Identification, Prentice Hall, New-Jersey, U.S.A.

13. R.K. PRASANTH 2011 IEEE Transactions on Signal Processing 59, pp. 3675-3684. State space system identification approach to radar data processing.

14. J. LARDIES and M.N TA 2011 Mechanical Systems and Signal Processing 25, pp.133150. Modal parameter identification of stay cables from output-only measurements.

15. L. ZHANG 2001 Proceedings of the $19^{\text {th }}$ IMAC, Orlando, Florida. Modal indicators for operational modal identification.

16. J.N. JUANG and R.S. PAPPA 1986 Journal of Guidance, Control and Dynamics 9, pp. 294-302. Effects of noise on modal parameters identified by the eigensystem realization algorithm.

17 S. DORVASH and S.N. PAKZAD 2012 Smart Materials and Structures 21, pp. 1-15. Effects of measurement noise on modal parameter identification.

18. T.-P. LE and P. ARGOUL 2015 Mechanical Systems and Signal Processing 52, pp. 2945. Distinction between harmonic and structural components in ambient excitation tests using the time-frequency domain decomposition technique.

19. G. DE PASQUALE and A.SOMA 2012 Proceedings of the $15^{\text {th }}$ DTIP, Cannes, France, Characterization of damping in vibrating microplates through measurement in the time domain. 


\section{List of Figures}

Figure 1:Normalized spectrum from the classical periodogram (a) $\mathrm{SNR}=3 \mathrm{~dB}$; (b) $\mathrm{SNR}=10 \mathrm{~dB}$

Figure 2: Normalized spectrum from the Welch estimator (a) $\mathrm{SNR}=3 \mathrm{~dB}$, (b) $\mathrm{SNR}=10 \mathrm{~dB}$

Figure 3: Spectrum of the iterative adaptive algorithm for the simulated case with $\mathrm{SNR}=10 \mathrm{~dB}$ ( a ) : one iteration; ( b ) : five iterations ; ( c ) : ten iterations

Figure 4: Contour plot spectrum of the iterative adaptive algorithm after ten iterations (a) $\mathrm{SNR}=3 \mathrm{~dB}$, (b) $\mathrm{SNR}=10 \mathrm{~dB}$

Figure 5: Stabilization diagram on eigenfrequencies and damping ratios for the simulated case

Figure 6: Schematic of the perforated microplate

Figure 7: Time response of the microplate to a step force actuation

Figure 8: Spectrum of the iterative adaptive algorithm for the experimental microplate ( a ) : one iteration; ( b ) : ten iterations

Figure 9: Contour plot spectrum of the iterative adaptive algorithm for the experimental microplate (after ten iterations)

Figure 10: Stabilization diagram on eigenfrequencies and damping ratios for the experimental microplate

Figure 11: Spectrum of the iterative adaptive algorithm for the experimental line cable ( a ) : one iteration; ( b ) : ten iterations

Figure 12: Contour plot spectrum of the iterative adaptive algorithm for the experimental line cable (after ten iterations)

Figure 13: Stabilization diagram on eigenfrequencies and damping ratios for the experimental line cable

Table 1. Outline of the iterative adaptive algorithm

Table 2. Natural frequencies and damping ratios of the line cable using the iterative adaptive algorithm (IAA) and the subspace method (SubEsp) 


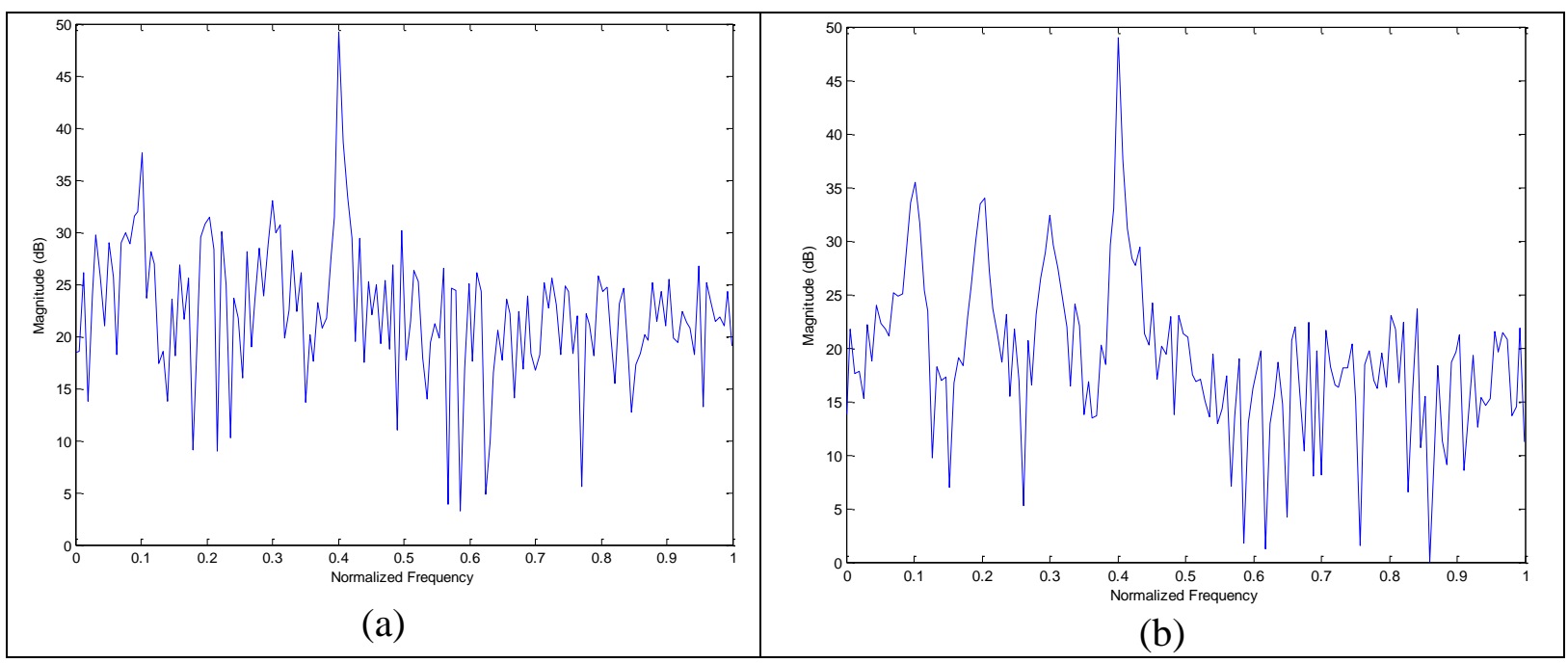

Figure 1: Normalized spectrum from the classical periodogram

(a) $\mathrm{SNR}=3 \mathrm{~dB}$; (b) $\mathrm{SNR}=10 \mathrm{~dB}$ 


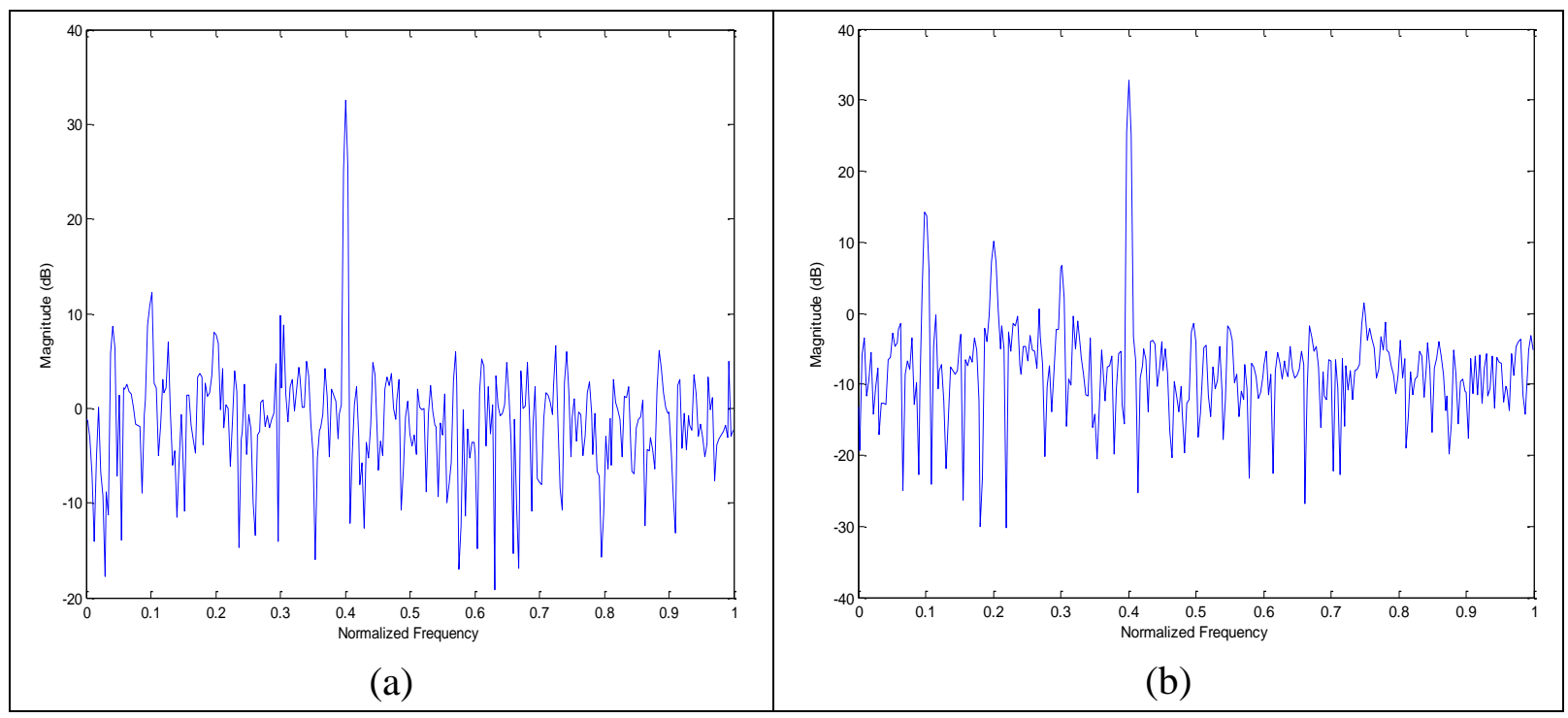

Figure 2: Normalized spectrum from the Welch estimator (a) $S N R=3 d B$, (b) $S N R=10 d B$ 


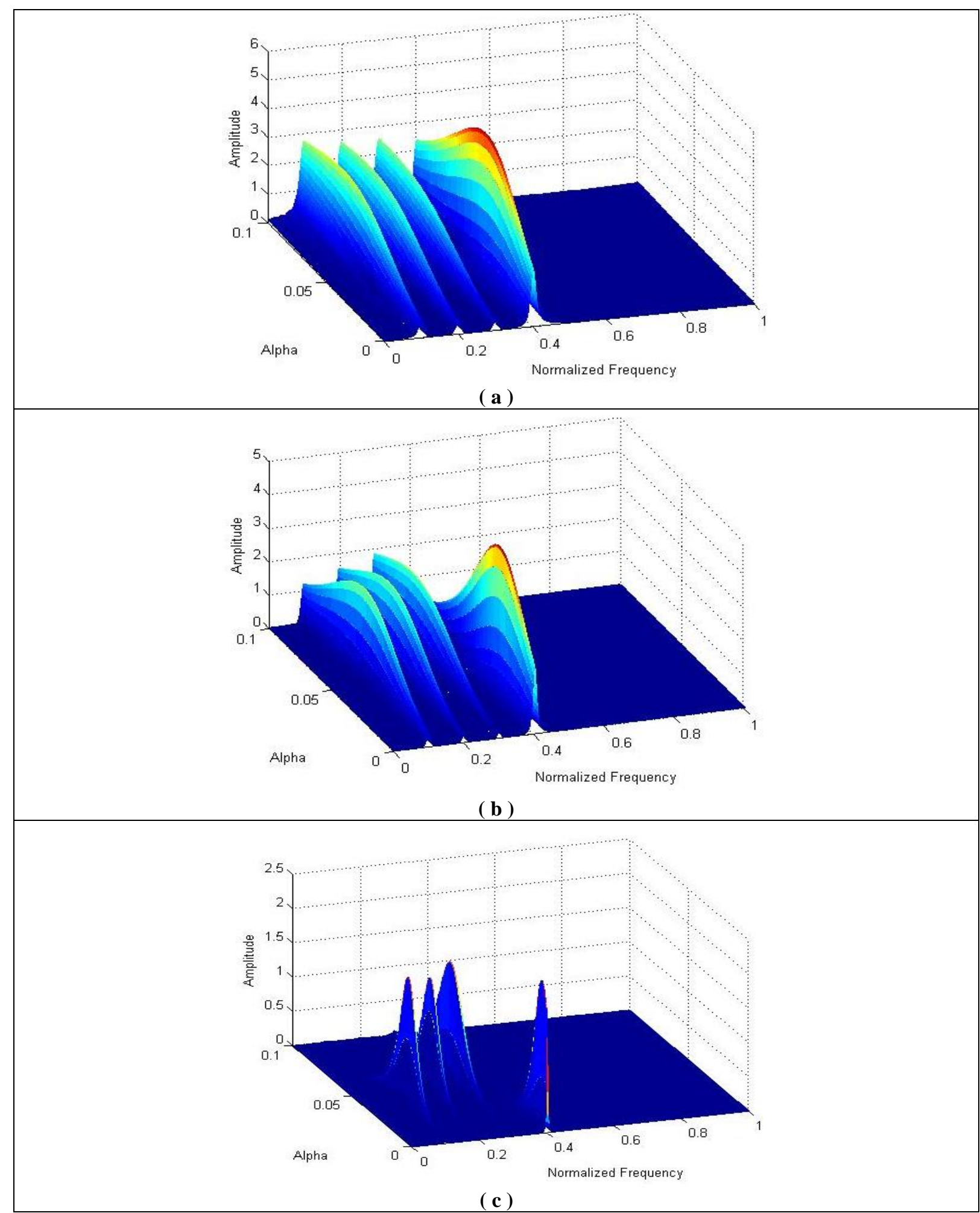

Figure 3: Spectrum of the iterative adaptive algorithm for the simulated case with $\mathrm{SNR}=10 \mathrm{~dB}$ ( a ) : one iteration; ( b ) : five iterations ; ( c ) : ten iterations 


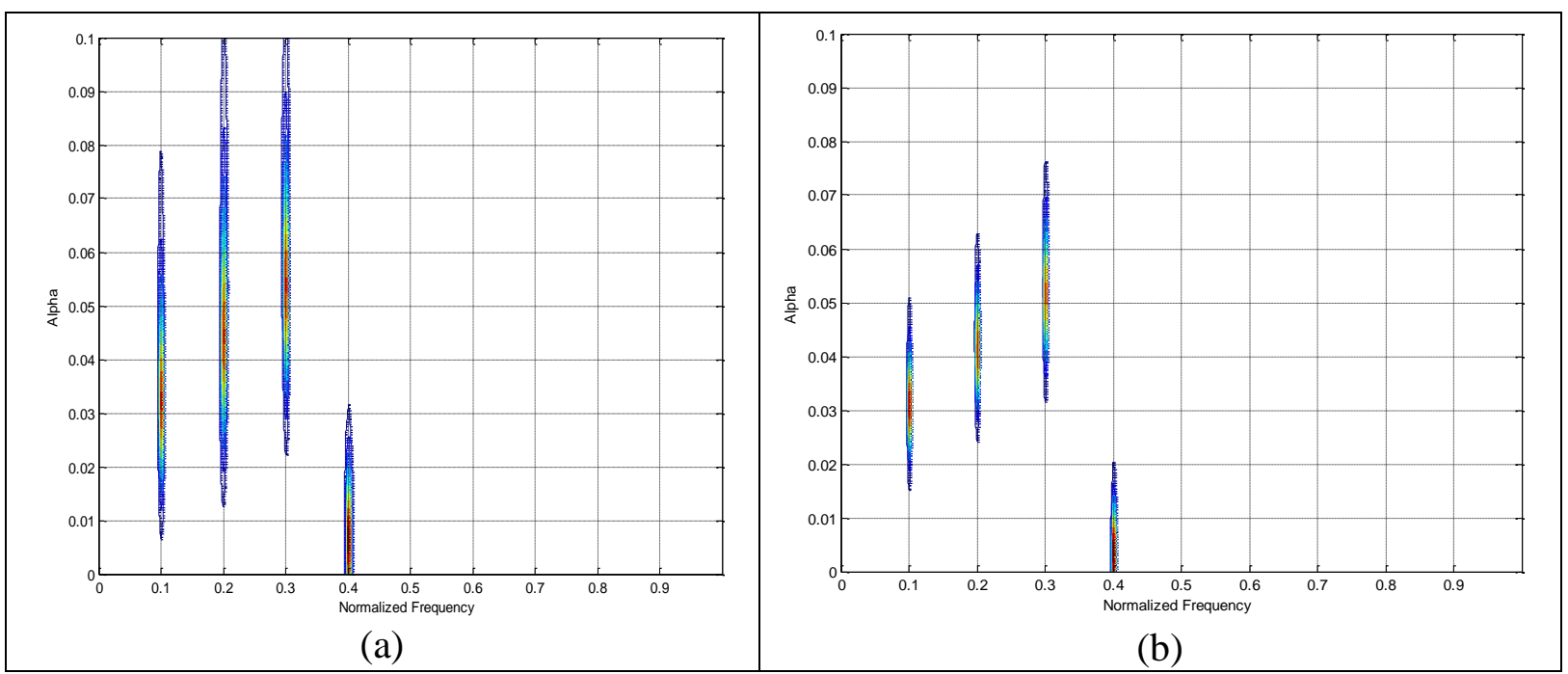

Figure 4: Contour plot spectrum of the iterative adaptive algorithm after ten iterations

(a) $\mathrm{SNR}=3 \mathrm{~dB}$, (b) $\mathrm{SNR}=10 \mathrm{~dB}$ 

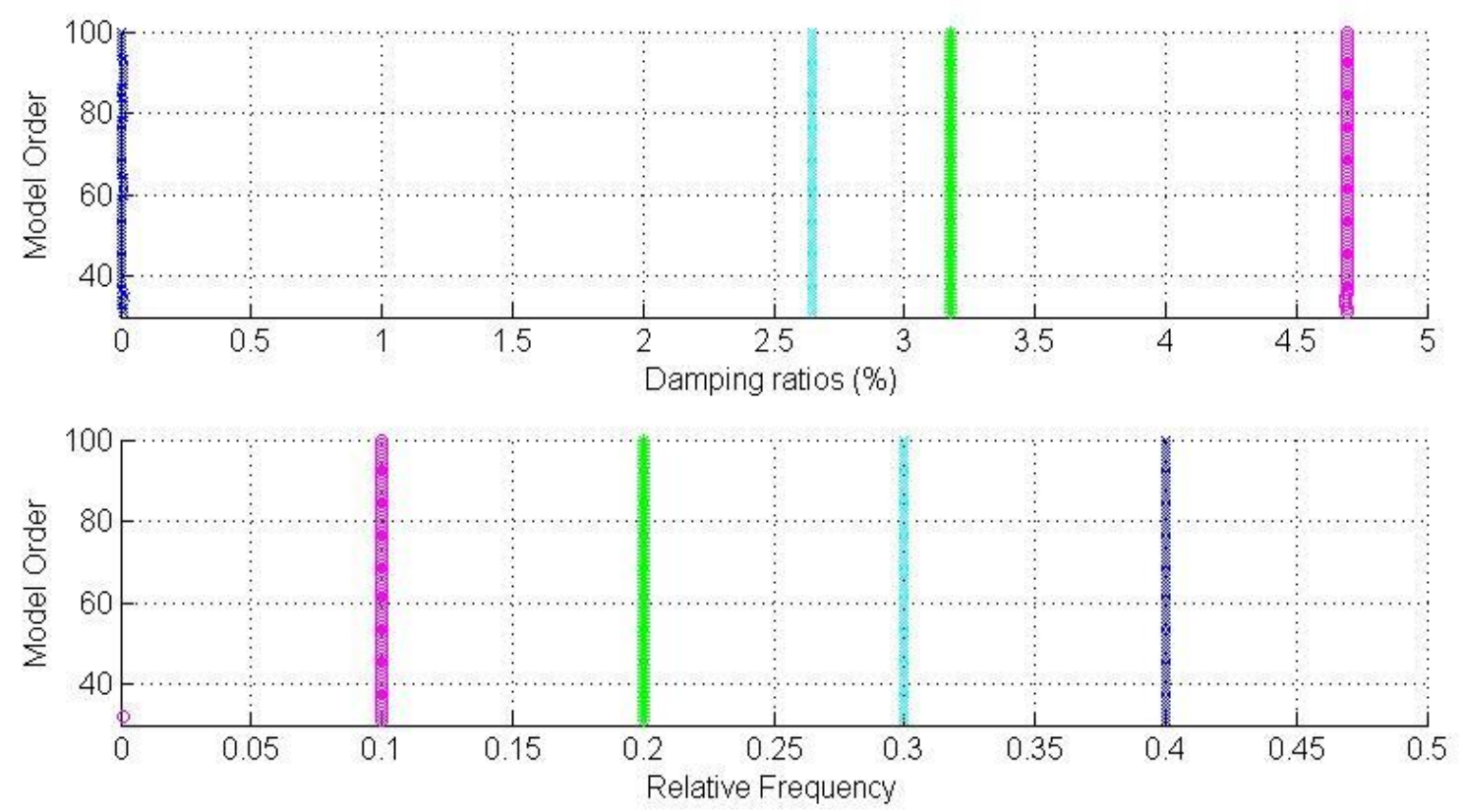

Figure 5: Stabilization diagram on eigenfrequencies and damping ratios for the simulated case 


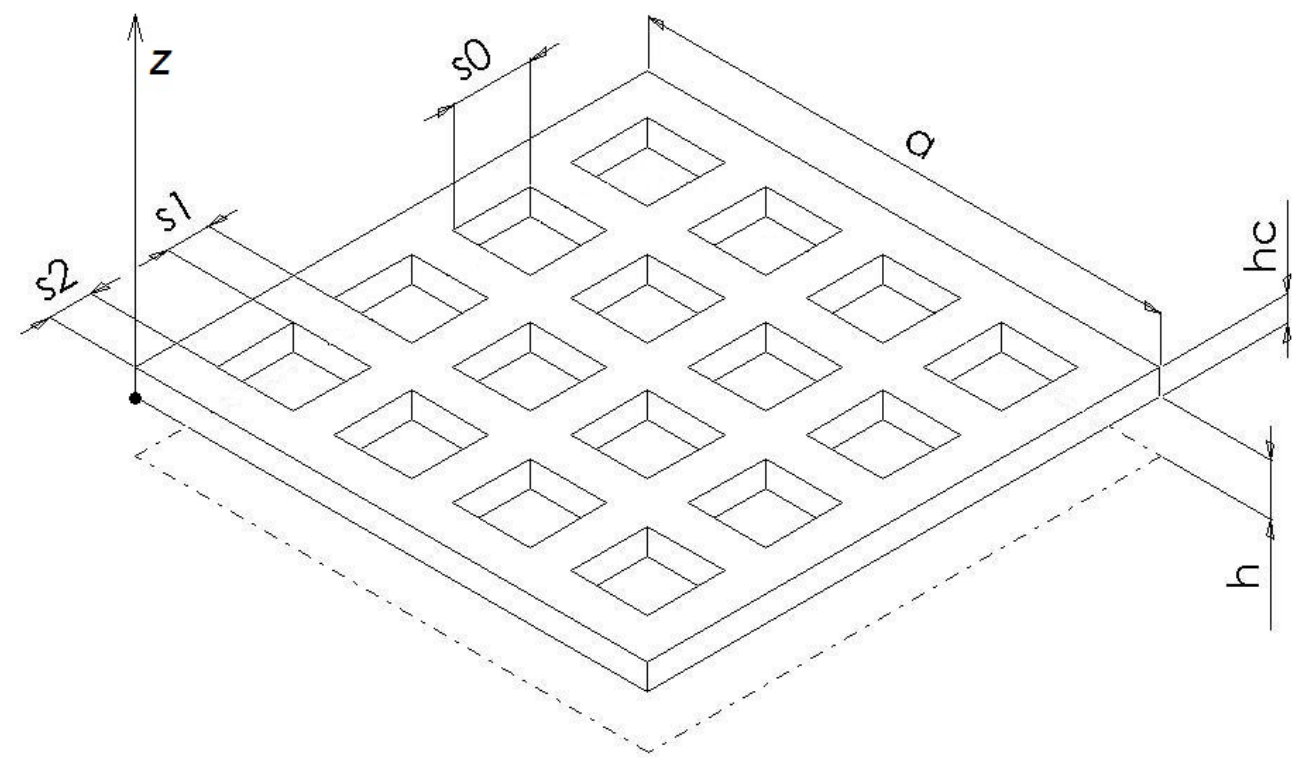

Figure 6: Schematic of the perforated microplate 


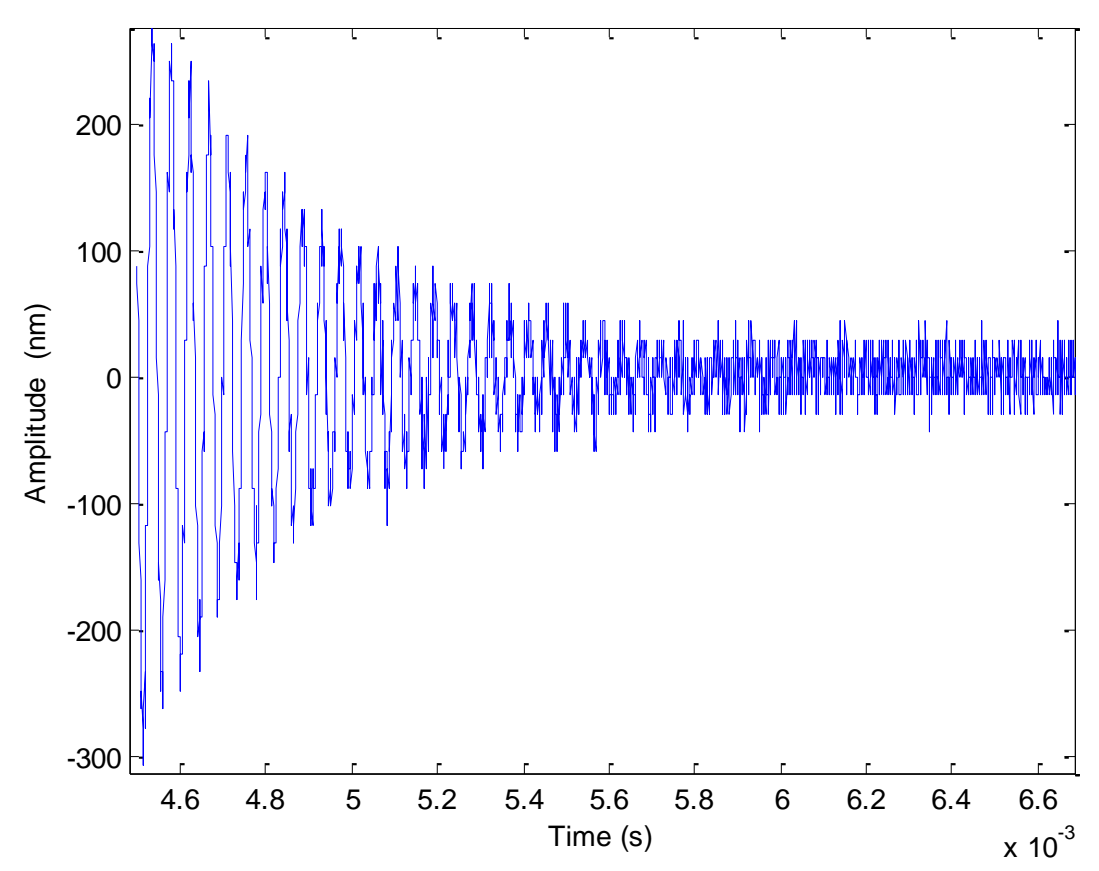

Figure 7: Time response of the microplate to a step force actuation 


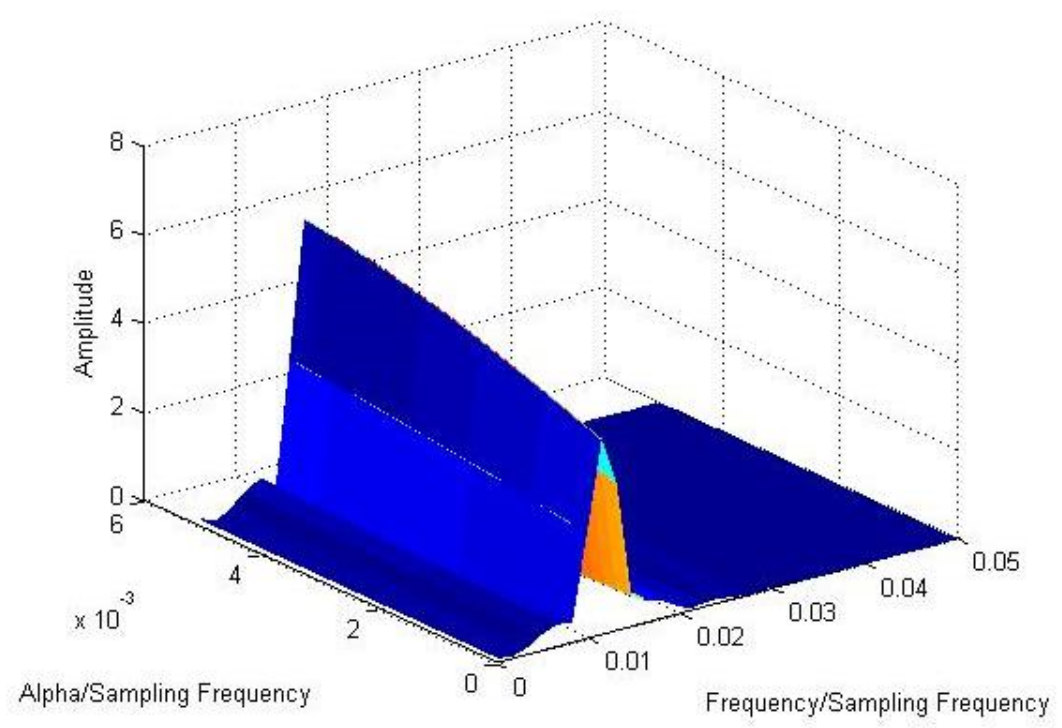

( a )

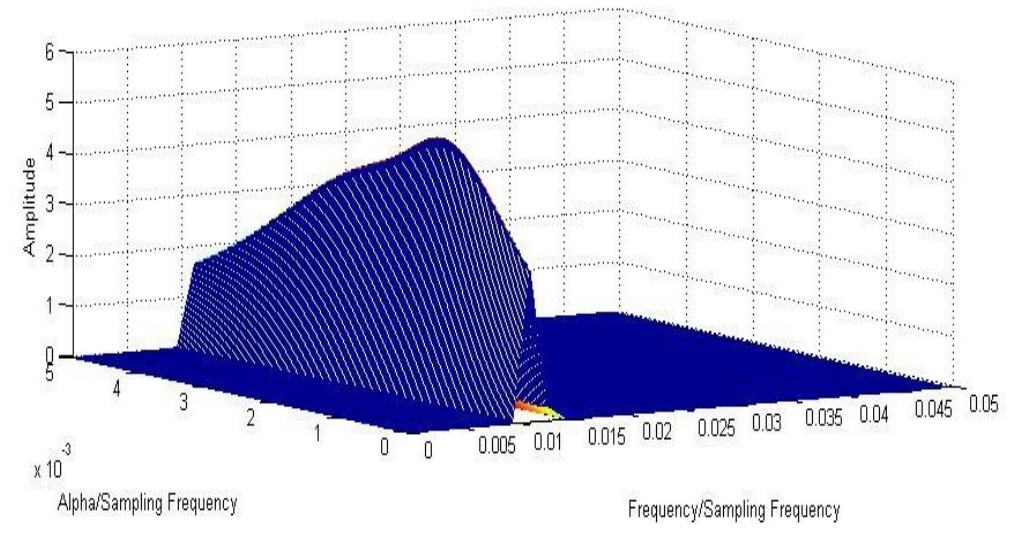

( b )

Figure 8: Spectrum of the iterative adaptive algorithm for the experimental microplate ( a ) : one iteration; ( b ) : ten iterations 


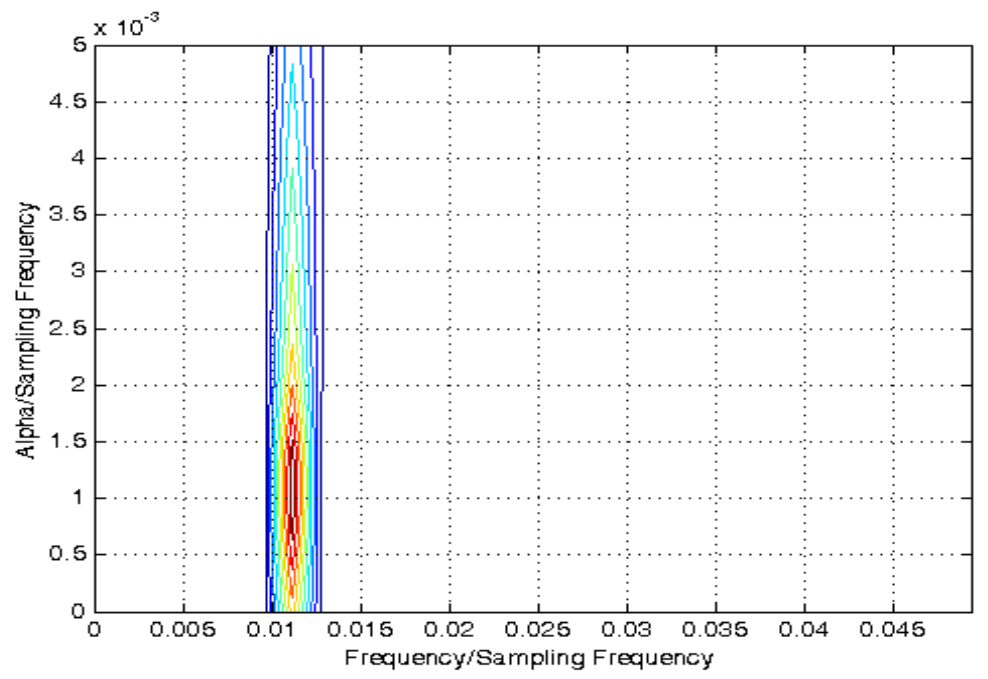

Figure 9: Contour plot spectrum of the iterative adaptive algorithm for the experimental microplate (after ten iterations) 

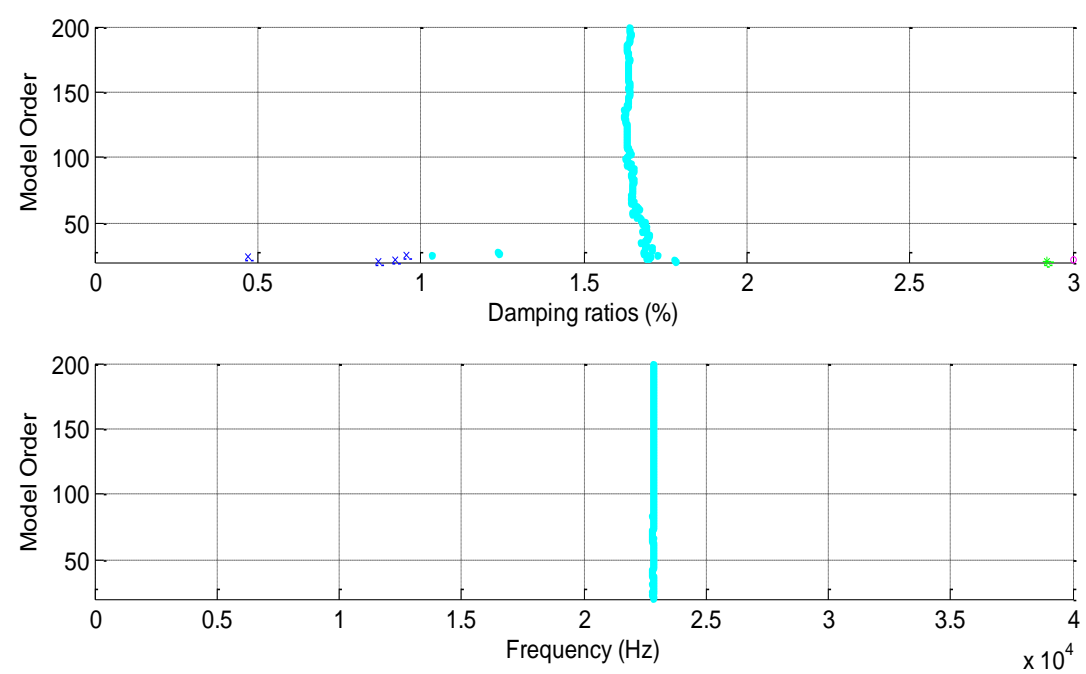

Figure 10: Stabilization diagram on eigenfrequencies and damping ratios for the experimental microplate 


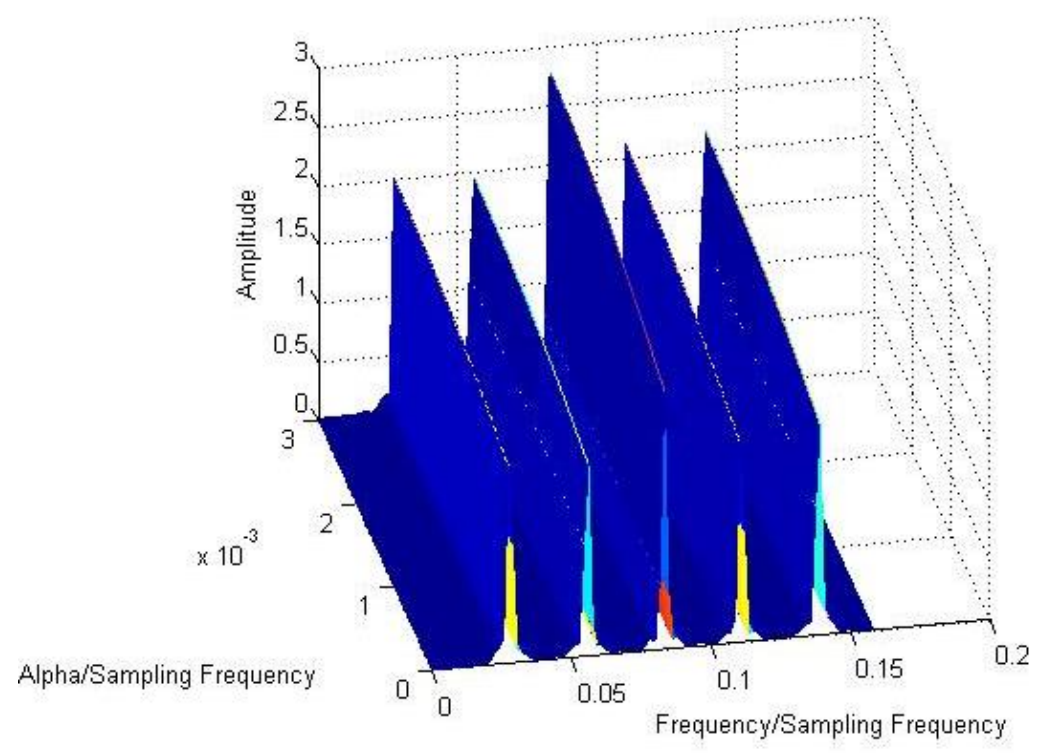

( a )

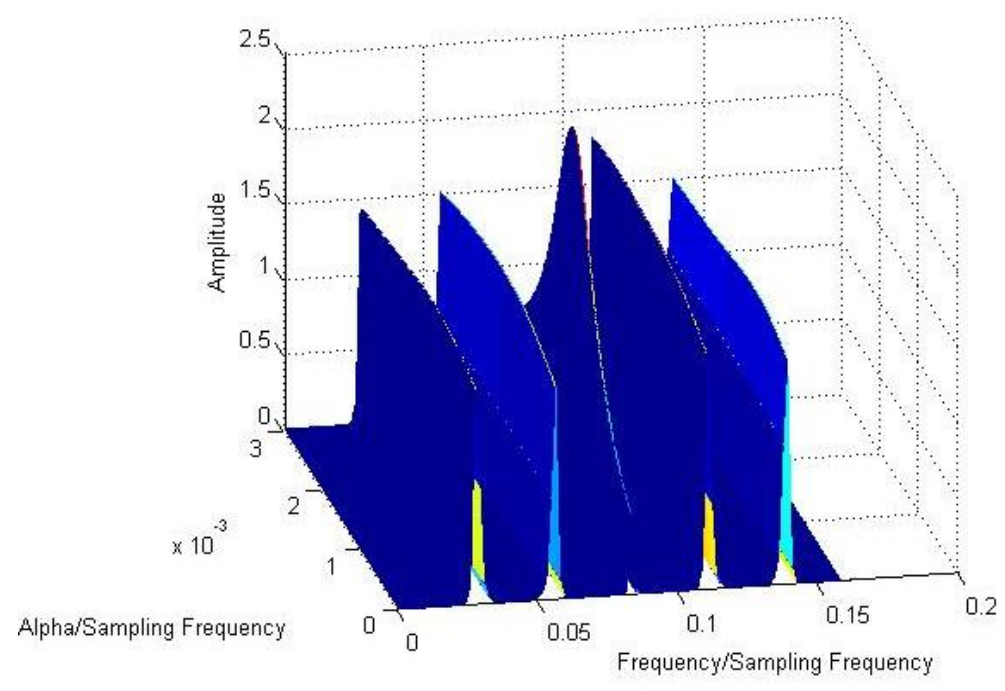

( b )

Figure 11: Spectrum of the iterative adaptive algorithm for the experimental line cable ( a ) : one iteration; ( b ) : ten iterations 


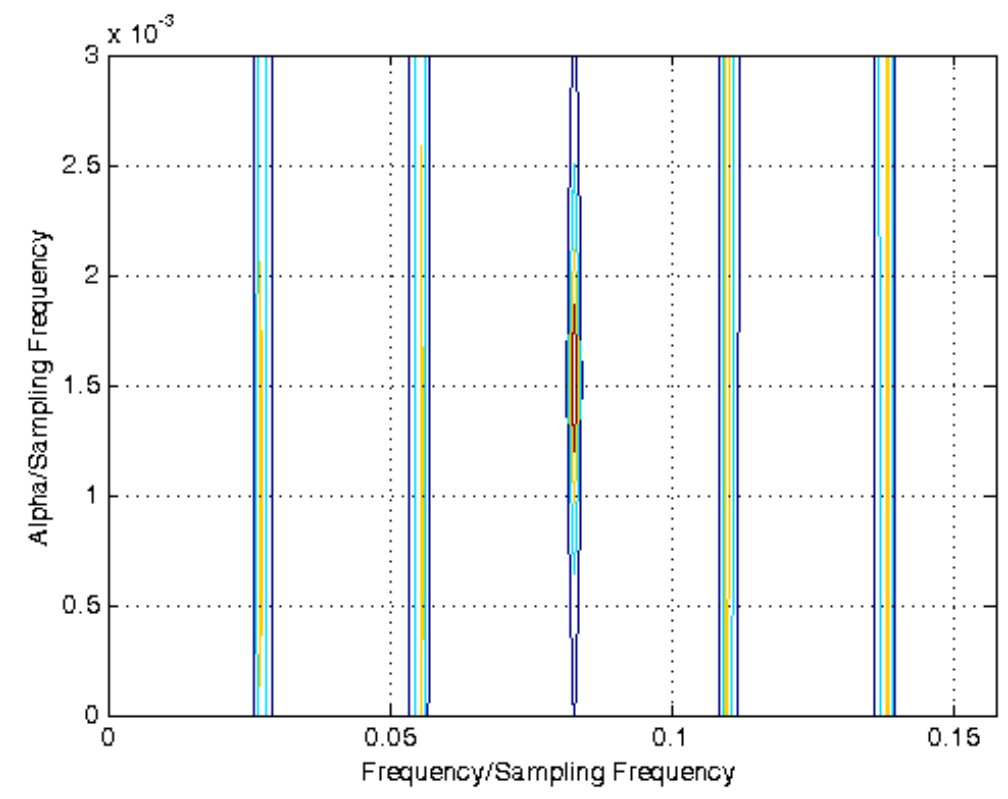

Figure 12: Contour plot spectrum of the iterative adaptive algorithm for the experimental line cable (after ten iterations) 

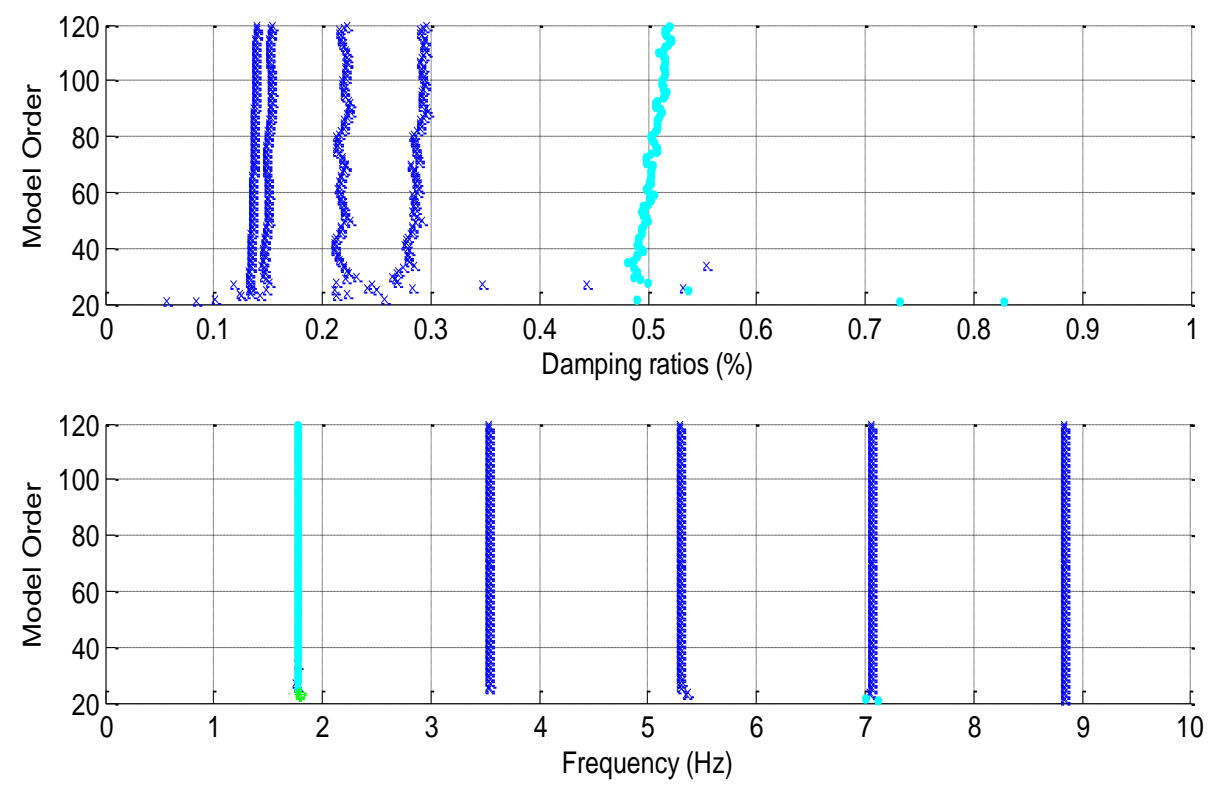

Figure 13: Stabilization diagram on eigenfrequencies and damping ratios for the experimental line cable 


\section{Initialisation}

Step 1 : Compute the initial estimate : $\quad \hat{B}_{k, m}^{(1)}=\frac{\boldsymbol{a}^{*} \boldsymbol{k}, \boldsymbol{m} \boldsymbol{y}}{\boldsymbol{a}_{\boldsymbol{k}, \boldsymbol{m}}^{*} \boldsymbol{a}_{\boldsymbol{k}, \boldsymbol{m}}}$

Step 2 : Compute the signal energy estimate at a grid point : $\quad \hat{p}_{k, m}^{(1)}=\left|\hat{B}_{k, m}^{(1)}\right|^{2}$

Step 3 : Compute the estimated covariance matrix $\boldsymbol{R}_{(I)}$ with the estimate $\hat{p}_{k, m}^{(1)}$ using (10)

\section{Iteration}

Step 4 : Compute the amplitude estimate at the ith iteration $\hat{B}_{k, m}^{(i)}=\frac{\boldsymbol{a}^{*} \boldsymbol{k}_{\boldsymbol{m}, \boldsymbol{m}} \boldsymbol{R}_{(\boldsymbol{i}-\boldsymbol{1})}^{-1} \boldsymbol{y}}{\boldsymbol{a}_{\boldsymbol{k}, \boldsymbol{m}}^{*} \boldsymbol{R}_{(\boldsymbol{i}-\mathbf{1})}^{-1} \boldsymbol{a}_{\boldsymbol{k}, \boldsymbol{m}}}$ with the most recent estimate of the covariance matrix

\section{Termination}

Step 5 : The iterative process will be stopped when the relative change in $\hat{B}_{k, m}$ between two iterations is : $\left|\hat{B}_{k, m}^{(i)}-\hat{B}_{k, m}^{(i-1)}\right|^{2}<10^{-3}$

Table 1. Outline of the iterative adaptive algorithm 


\begin{tabular}{|c|c|c|c|c|c|}
\hline Modes & 1 & 2 & 3 & 4 & 5 \\
\hline$F_{\text {Theo }}(\mathrm{Hz})$ & 1.765 & 3.530 & 5.295 & 7.060 & 8.825 \\
\hline$F_{\text {IAA }}(\mathrm{Hz})$ & 1.742 & 3.516 & 5.247 & 6.981 & 8.741 \\
\hline F $_{\text {SubEsp }}(\mathrm{Hz})$ & 1.771 & 3.521 & 5.248 & 7.121 & 8.795 \\
\hline$\xi_{\text {IAA }}(\%)$ & 0.507 & 0.231 & 0.304 & 0.148 & 0.166 \\
\hline$\xi_{\text {SubEsp }}(\%)$, & 0.511 & 0.218 & 0.289 & 0.138 & 0.153 \\
\hline
\end{tabular}

Table 2. Natural frequencies and damping ratios of the line cable using the iterative adaptive algorithm (IAA) and the subspace method (SubEsp) 